\title{
Estimation of Earthquake Hazard Parameters from Incomplete Data Files. Part III. Incorporation of Uncertainty of Earthquake-Occurrence Model
}

\author{
by Andrzej Kijko, Ansie Smit, and Markvard A. Sellevoll
}

\begin{abstract}
Most probabilistic seismic-hazard analysis procedures require that at least three seismic source parameters be known, namely the mean seismic activity rate $\lambda$, the Gutenberg-Richter $b$-value, and the area-characteristic (seismogenic source) maximum possible earthquake magnitude $m_{\max }$. In almost all currently used seismic-hazard assessment procedures that utilize these three parameters, it is explicitly assumed that all three remain constant over time and space. However, closer examination of most earthquake catalogs has indicated that significant spatial and temporal variations existed in the seismic activity rate $\lambda$, as well as in the Gutenberg-Richter $b$-value. In this study, the maximum likelihood estimation of these earthquake hazard parameters considers the incompleteness of the catalogs, the uncertainty in the earthquake magnitude determination, as well as the uncertainty associated with the applied earthquake-occurrence models. The uncertainty in the earthquake-occurrence models is introduced by assuming that both the mean seismic activity rate $\lambda$ and the Gutenberg-Richter $b$-value are random variables, each described by the gamma distribution. This approach results in the extension of the classic frequency-magnitude Gutenberg-Richter relation and the Poisson distribution of the number of earthquakes with their compounded counterparts (Benjamin, 1968; Campbell, 1982, 1983). The proposed procedure was applied in the estimation of the seismicity parameters in an area that had experienced the strongest and most devastating earthquake in contemporary South African history, namely the 29 September $1969 M_{\mathrm{w}} 6.3$ Ceres-Tulbagh event. In this example, it was shown that the introduction of uncertainty in the earthquake-occurrence model reduced the mean return periods, leading to an increase of the estimated seismic hazard. Additionally, this study confirmed that accounting for magnitude uncertainties had the opposite effect, that is, it brought about increases in the return periods, or, equivalently, a reduction of the estimated seismic hazard.
\end{abstract}

\section{Introduction}

The problem of incomplete seismic-event catalogs is often encountered in the analysis of seismic hazard. The significant aspects that influence the completeness of earthquake catalogs include the historical and socioeconomic context and the demographic variations and alterations in the seismic network. In most instances, the degree of completeness is a monotonically increasing function of time, where the more recent portion of the catalog is more complete than the older part. In this study, we propose a methodology for the assessment of the key seismic-hazard parameters in the form of the mean seismic activity $\lambda$, the $b$-value of the frequencymagnitude Gutenberg-Richter relation, and $m_{\max }$, the areacharacteristic maximum possible earthquake magnitude. The proposed methodology extends the procedures by Kijko and Sellevoll $(1989,1992)$ to include the instance where the uncertainty of the earthquake-occurrence model is taken into account. Henceforth, the Kijko and Sellevoll procedures are, respectively, referred to as KS-I (Kijko and Sellevoll, 1989) and KS-II (Kijko and Sellevoll, 1992).

Both KS-I and KS-II provide for the incompleteness of the earthquake event catalogs by accounting for historical (largest event only) and instrumental complete records with different levels of completeness. KS-II also accounts for the uncertainty in earthquake magnitude determination.

Figure 1 shows a typical scenario encountered when conducting area-characteristic seismic-hazard assessments, based on historical and complete catalogs. It is assumed that the complete part of the catalog can be divided into $s$ subcatalogs $(i=1, \ldots, s)$. Each of these subcatalogs is considered to be complete for event magnitudes exceeding a certain magnitude level $m_{\min }^{(i)}$, during a certain period of time $t_{i}$. The magnitude $m_{\min }^{(i)}$ denotes the level of completeness for each 


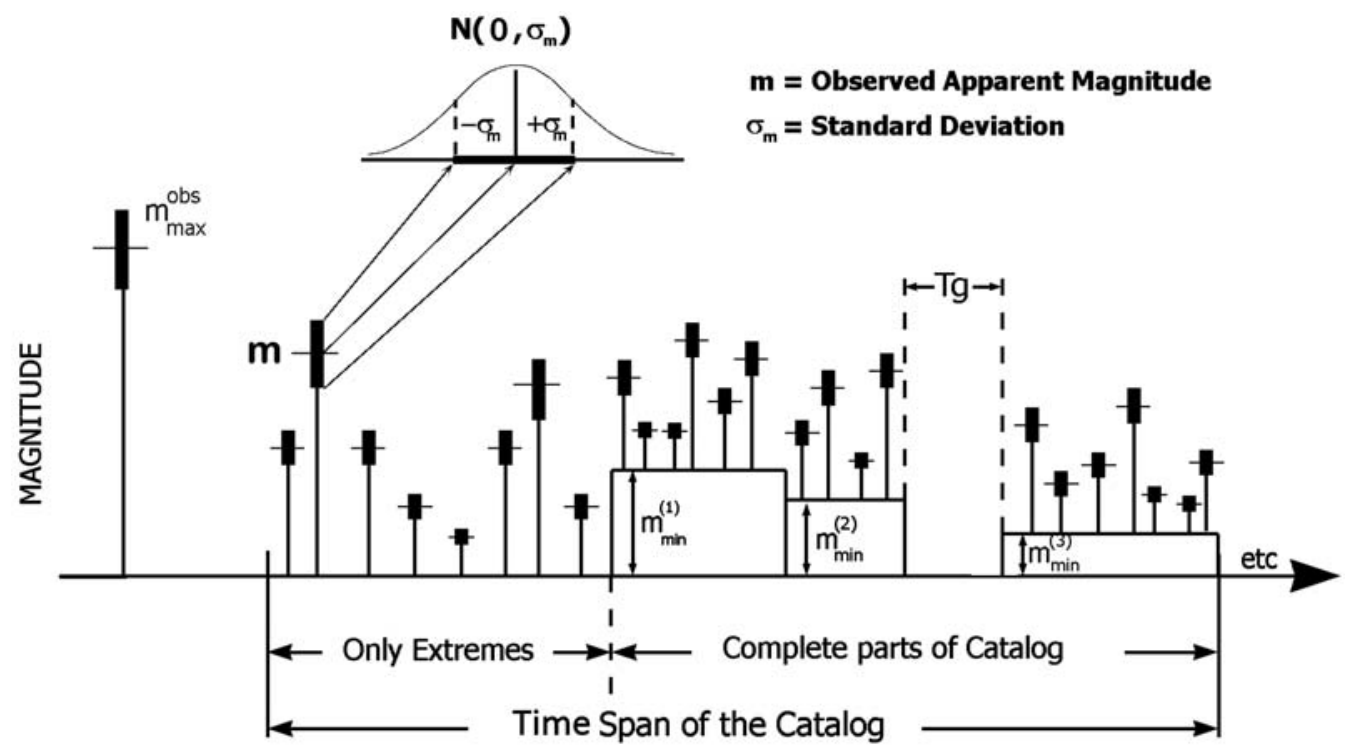

Figure 1. The data that can be used to obtain basic seismic-hazard parameters for the area in the vicinity of the selected site by the procedure used. The approach permits the combination of largest earthquake data and complete data that have variable threshold magnitudes. It allows the use of the largest known historical earthquake $\left(m_{\max }^{\text {obs }}\right)$, which occurred before the start of the catalog. It also accepts gaps $\left(T_{g}\right)$ when records are missing or the seismic networks are nonoperational. Uncertainty in earthquake magnitude is also taken into account, in that an assumption is made that the observed magnitude is the true magnitude subjected to a random error, which follows a Gaussian distribution, having a zero mean and a known standard deviation (after Kijko and Sellevoll, 1992 [KS-II]).

subcatalog. This approach permits the occurrence of gaps $\left(T_{g}\right)$ to account for missing event records. Missing records could be caused by, for example, seismic networks being nonoperational. The KS-II procedure can also account for uncertainties in determining seismic-event magnitudes by considering the assumption that the observed magnitude is the true magnitude, subject to a random error. It is assumed that this random error follows a Gaussian distribution, having a zero mean and a known standard deviation (Tinti and $\mathrm{Mu}-$ largia, 1985; McGuire, 2004; Márquez-Ramírez et al., 2015).

In addition to providing for incompleteness and the magnitude uncertainty in earthquake catalogs, the ultimate methodology for earthquake hazard assessment should also consider the inevitable discrepancy between the data and the applied models describing the earthquake occurrence. The statistical tools applied in the development of such methodology are described in the following section.

\section{Earthquake-Occurrence Model}

The classic assumption about the temporal distribution of the number of earthquakes within a specified area is that it can be described by a Poisson process (Cornell, 1968; Lomnitz, 1973; Gardner and Knopoff, 1974). Therefore, the probability that a total of $n$ earthquakes will be observed during the specified time interval $t$ within a specified area is described as

$$
P_{n}(\lambda, t)=\frac{(\lambda t)^{n}}{n !} \exp (-\lambda t), \quad n=0,1,2 \ldots
$$

in which $\lambda \equiv \lambda\left(m_{\min }\right)$ refers to the parameter of the Poisson distribution and describes the mean (usually annual) areacharacteristic activity rate of earthquake occurrence, with the magnitude equal to or greater than $m_{\min }$.

Based on observations, it is commonly assumed that the distribution of the number of seismic events, with respect to their magnitude, follows the classic Gutenberg-Richter relation (Gutenberg and Richter, 1942, 1956)

$$
\log N(m)=a-b m,
$$

in which $N(m)$ is the number of seismic events with magnitude $m \geq m_{\min }$ occurring within a specified period of time. Coefficients $a$ and $b$ depend on the tectonic features of the relevant seismogenic source. Equation (2) is equivalent to the assumption that the cumulative distribution function (CDF) of seismic-event magnitude is of the form (Aki, 1965):

$$
F_{M}(m) \equiv P(M \leq m)=1-\exp \left(-\beta\left(m-m_{\min }\right)\right),
$$

for $m \geq m_{\min }$ and $\beta=b \ln (10)$.

An explicit assumption relevant to most seismic-hazard assessment procedures, as indicated by authors such as Cornell (1968) and McGuire $(1976,1978)$, is that the parameters $\lambda$ and $b$ remain constant over time. However, closer examination of most seismic-event catalogs has indicated that there were temporal changes in both these parameters. For some seismic areas, the $b$-value has been reported to increase or decrease before the large seismic events. Usually, such changes are explained by the state of the stress, namely the higher the stress level, the lower the $b$-value (Gibowicz 
and Kijko, 1994; Sharma et al., 2013; Scholz, 2015). Other theories link the $b$-value with the homogeneity of the rock, namely the more heterogeneous the rock, the higher the $b$-value (Wyss et al., 1997; Wesseloo, 2014; Wesseloo et al., 2014). Regardless of the mechanism, the space-time fluctuation of the $b$-value is a well-known phenomenon. A wide range of opinions concerning changes of patterns in seismicity, together with those mentioned above, as well as reference lists, are available in a monograph by Simpson and Richards (1981). In addition, the aforementioned are also available in two special issues of Wyss et al. (1999) and Mora et al. (2001). A list of well-documented instances of the temporal variation of seismic activity worldwide is also provided by Cornell and Winterstein (1988), Ogata and Abe (1991), Kijko and Graham (1998), Karakaisis et al. (2002), and more recently Talbi and Yamazaki (2009).

One of the easiest ways to account for fluctuations in the $\lambda$ and $b$ parameters is by introducing compound distributions. Compound distributions (sometimes referred to as Bayesian distributions) provide a tool that accounts for the instance in which a parameter, in the distribution of a random variable, is also a random variable (DeGroot, 1970). These distributions are used in many probabilistic models applied in engineering (Hamada et al., 2008), as well as in the insurance and risk industry (Klugman et al., 2008). To our knowledge, the first authors to apply compound distributions in seismic-hazard assessment were Benjamin (1968) and Campbell (1982, 1983).

When compound distributions are utilized to account for the uncertainty in $\lambda$ and $b$, the seismic parameters are treated as random variables that are distributed according to certain specified distributions; for example, the gamma distribution. The gamma distribution is used extensively to model various random variables, as it does not impose too many limitations, and it can fit a large variety of shapes. The probability density function (PDF) of gamma distribution is defined as (Abramowitz and Stegun, 1970)

$$
f_{X}(x)=x^{(q-1)} \frac{p^{q}}{\Gamma(q)} \exp (-p x) \quad x, p, q>0,
$$

in which $\Gamma(q)$ is the gamma function:

$$
\Gamma(q)=\int_{0}^{\infty} y^{q-1} \exp (-y) d y \quad q>0 .
$$

The distribution parameters $p$ and $q$ are related to the mean $\mu_{x}$ and the variance $\sigma_{x}^{2}$ of the distribution according to

$$
\begin{gathered}
\mu_{x}=\frac{q}{p}, \\
\sigma_{x}^{2}=\frac{q}{p^{2}} .
\end{gathered}
$$

The coefficient of variation expresses the uncertainty related to a given parameter, in this instance the variation of a param- eter relative to its mean. A higher value indicates a greater dispersion of the parameter, and is given by

$$
v_{x}=\frac{\sigma_{x}}{\mu_{x}} .
$$

The Poisson distribution (1) is combined with the gamma distribution (4) to create the Poisson-gamma compound distribution to obtain the probability to observe $n$ seismic events, within a time interval $t$, for temporal varying seismic activity $\lambda$ (Benjamin, 1968), as follows:

$$
\begin{aligned}
P_{n}\left(\bar{\lambda}, t, v_{\lambda}\right) & =\int_{0}^{\infty} P_{n}(\lambda, t) f_{\Lambda}(\lambda) d \lambda \\
& =\frac{\Gamma\left(n+q_{\lambda}\right)}{n ! \Gamma\left(q_{\lambda}\right)}\left(\frac{p_{\lambda}}{t+p_{\lambda}}\right)^{q_{\lambda}}\left(\frac{t}{t+p_{\lambda}}\right)^{n},
\end{aligned}
$$

in which $\Gamma(n)$ is the gamma function (5), $p_{\lambda}$ and $q_{\lambda}$ are the parameters of gamma distribution (4), such that $p_{\lambda}=\bar{\lambda} / \sigma_{\lambda}^{2}$, $q_{\lambda} \equiv \nu_{\lambda}^{(-2)}=\bar{\lambda}^{2} / \sigma_{\lambda}^{2}$, and $\bar{\lambda}$ denotes the mean value of the activity rate $\lambda$.

The compound CDF of earthquake magnitudes is derived in a similar way by combining the classic Gutenberg-Richter earthquake magnitude distribution (3) and the probability density gamma distribution of $\beta$ (4). The resulting compound Gutenberg-Richter CDF of earthquake magnitudes is subsequently normalized with the introduction of an upper limit of the earthquake magnitude $m_{\max }$, and it takes the form of the exponential-gamma distribution, as described by Campbell (1982):

$$
F_{M}\left(m \mid v_{\beta}, m_{\min }\right)=C_{\beta}\left[1-\left(\frac{p_{\beta}}{p_{\beta}+m-m_{\min }}\right)^{q_{\beta}}\right],
$$

in which $p_{\beta}=\bar{\beta} /\left(\sigma_{\beta}^{2}\right)$ and $q_{\beta} \equiv\left(v_{\beta}\right)^{-2}=\bar{\beta}^{2} / \sigma_{\beta}^{2}$, such that $\bar{\beta}$ denotes the mean value of parameter $\beta$, and $\sigma_{\beta}$ refers to the standard deviation of $\bar{\beta}$. The normalizing coefficient $C_{\beta}$ is defined as

$$
C_{\beta}=\left[1-\left(\frac{p_{\beta}}{p_{\beta}+m_{\max }-m_{\min }}\right)^{q_{\beta}}\right]^{-1} .
$$

As $q_{\lambda}=\bar{\lambda} p_{\lambda}$ and $q_{\beta}=\bar{\beta} p_{\beta}$, the two compound distributions (9) and (10) may, respectively, be written in alternative forms as

$$
P_{n}\left(\bar{\lambda}, t, v_{\lambda}\right)=\frac{\Gamma\left(n+q_{\lambda}\right)}{n ! \Gamma\left(q_{\lambda}\right)}\left(\frac{q_{\lambda}}{\bar{\lambda} t+q_{\lambda}}\right){ }^{q_{\lambda}}\left(\frac{\bar{\lambda} t}{\bar{\lambda} t+q_{\lambda}}\right),
$$

and

$$
F_{M}\left(m \mid v_{\beta}, m_{\min }\right)=C_{\beta}\left[1-\left(\frac{q_{\beta}}{q_{\beta}+\bar{\beta}\left(m-m_{\min }\right)}\right)^{q_{\beta}}\right],
$$

in which 


$$
C_{\beta}=\left[1-\left(\frac{q_{\beta}}{q_{\beta}+\bar{\beta}\left(m_{\max }-m_{\min }\right)}\right)^{q_{\beta}}\right]^{-1},
$$

and the parameters $\bar{\lambda}$ and $\bar{\beta}$ are estimated by applying the maximum likelihood procedure.

Based on (13) and (14), the associated compound PDF of earthquake magnitudes are defined as

$$
f_{M}\left(m \mid v_{\beta}, m_{\min }\right)=C_{\beta} \bar{\beta}\left(\frac{q_{\beta}}{q_{\beta}+\bar{\beta}\left(m-m_{\min }\right)}\right)^{q_{\beta}+1} .
$$

The proposed implementation of the compound distributions is similar to procedures described by Benjamin (1968) and Campbell $(1982,1983)$, with the two applications only differing in the parameterization of the variables. The new proposed methodology to account for the uncertainty for hazard parameters or, equivalently, the account for the aleatory uncertainty of the earthquake-occurrence model, is anything but unique. Possibly, the most frequently applied alternative approach is based on the more complex Bayesian formalisms, as applied by Pisarenko et al. (1996), Pisarenko and Lyubushin (1997), Ogata (1999), and Rotondi and Varini (2007). Shi and Bolt (1982) and Guttorop and Hopkins (1986) also addressed the question of fluctuations in the hazard parameters by applying an approximate variance stabilizing transformation to the $b$-value, which is assumed to be a slowly varying random variable.

\section{Parameter Estimation}

The sought area-characteristic seismic-hazard parameters $\boldsymbol{\theta}=\left(\bar{\lambda}, \bar{\beta}, m_{\max }\right)$ are estimated by the maximum likelihood method.

\section{Historical Earthquakes}

The likelihood functions for historical seismic catalogs are based only on the strongest observed events; therefore, the extreme magnitude compound distribution is used. Assume that the historical part of the catalog with a time span of $t_{0}$ contains $n_{0}$ largest seismic events, each with a magnitude equal to or exceeding a certain magnitude value $m_{0}$, such that $m_{0}$ is larger or equal to the overall minimum magnitude of interest for the whole catalog $m_{\min }$. The only restriction on the choice of this value is that $m_{\min }$ should not exceed the threshold magnitude of any part (historical or complete) of the catalog. The time span of the historical part of the catalog $t_{0}$ can be expressed as the sum of the time intervals $t_{0 k}$ between the historical events for $k=1, \ldots, n_{0}$.

The probability that in an arbitrary time interval $t$ either no earthquake occurs or all occurring earthquakes have magnitudes not exceeding $m_{0}$ (Epstein and Lomnitz, 1966; Gan and Tung, 1983; Gibowicz and Kijko, 1994), could be expressed as follows

$$
F_{M}^{\max }\left(m \mid v_{\beta}, m_{0}, t\right)=\sum_{n=0}^{\infty} P_{n}\left(\bar{\lambda}, t, v_{\lambda}\right)\left[F_{M}\left(m \mid v_{\beta}, m_{0}\right)\right]^{n},
$$

or alternatively (Campbell, 1982) as follows:

$$
F_{M}^{\max }\left(m \mid v_{\beta}, m_{0}, t\right)=\left[\frac{q_{\lambda}}{q_{\lambda}+\bar{\lambda}_{0} t\left[1-F_{M}\left(m \mid v_{\beta}, m_{0}\right)\right.}\right]^{q_{\lambda}},
$$

by applying the theorem of total probability (Cramér, 1961). It follows from (17) that the PDF of the largest seismic-event magnitude within the time period $t$ is

$$
f_{M}^{\max }\left(m \mid v_{\beta}, m_{0}, t\right)=\frac{\bar{\lambda}_{0} t q_{\lambda} f_{M}\left(m \mid v_{\beta}, m_{0}\right) F_{M}^{\max }\left(m \mid v_{\beta}, m_{0}, t\right)}{q_{\lambda}+\bar{\lambda}_{0} t\left[1-F_{M}\left(m \mid v_{\beta}, m_{0}\right)\right]},
$$

in which $\bar{\lambda}_{0}=\bar{\lambda}\left[1-F_{M}\left(m \mid v_{\beta}, m_{0}\right)\right]$ denotes the mean (usually annual) activity rate for seismic events with magnitudes $m \geq m_{0}$, and $\bar{\lambda} \equiv \bar{\lambda}\left(m_{\min }\right)$ is the mean activity rate corresponding to the magnitude value $m_{\min }$. The functions $F_{M}\left(m \mid v_{\beta}, m_{0}\right)$ and $f_{M}\left(m \mid v_{\beta}, m_{0}\right)$, respectively, denote the CDF and PDF of the seismic-event magnitude, described by (13) and (15).

Based on (18), the subsequent sample likelihood function of the unknown parameters for the historical events takes the form:

$$
L_{H}\left(\boldsymbol{\theta} \mid \mathbf{m}_{0}, \mathbf{t}_{0}, \mathbf{v}\right) \equiv L_{H}(\boldsymbol{\theta})=\prod_{k=1}^{n_{0}} f_{M}^{\max }\left(m_{0 k} \mid v_{\beta}, m_{0}, t_{0 k}\right),
$$

in which vectors $\mathbf{m}_{0}$ and $\mathbf{t}_{0}$ are, respectively, defined as the $\left(n_{0} \times 1\right)$ vectors, consisting of the $n_{0}$ largest earthquake magnitudes $m_{0 k}$, associated with time intervals $t_{0 k}$, when $k=1, \ldots, n_{0}$. The vector $\mathbf{v}=\left(v_{\lambda}, v_{\beta}\right)$ consists of the coefficients of variation of the unknown $\bar{\lambda}$ and $\bar{\beta}$.

\section{Complete Catalogs with Different Levels of Completeness}

Assume that the second, complete part of the catalog can be divided into $s$ subcatalogs (Fig. 1). Each subcatalog has a time span $t_{i}(i=1, \ldots, s)$ and is complete, starting from the known magnitude $m_{\min }^{(i)}$. For each subcatalog $i$, the vector $\mathbf{m}_{i}$ denotes $n_{i}$ observed seismic-event magnitudes $m_{i k}$, such that $m_{i k} \geq m_{\min }^{(i)}$, in which $k=1, \ldots, n_{i}$. Let $\mathbf{L}_{i}\left(\boldsymbol{\theta} \mid \mathbf{m}_{i}\right)$ denote the likelihood function of the unknown $\boldsymbol{\theta}=\left(\bar{\lambda}, \overline{\boldsymbol{\beta}}, m_{\max }\right)$, based on the $i$ th complete subcatalog. If the magnitude of the seismic events is independent of their number, the likelihood function $L_{\mathrm{i}}\left(\boldsymbol{\theta} \mid \mathbf{m}_{i}, t_{i}\right)$ of each of complete subcatalog $i$ is the product of the two functions: $L_{\mathrm{i}}\left(\bar{\lambda} \mid n_{i}, t_{i}\right)$ the likelihood function of $\bar{\lambda}$, and $L_{i}\left(\bar{\beta} \mid \mathbf{m}_{i}\right)$ the likelihood function of $\bar{\beta}$.

The assumption that the number of seismic events per unit time is distributed according to the compound Poisson-gamma 
distribution (12) means that for each complete subcatalog, the likelihood function $L_{\mathrm{i}}\left(\bar{\lambda} \mid n_{\mathrm{i}}, t_{\mathrm{i}}\right)$ has the form:

$$
L_{i}\left(\bar{\lambda} \mid n_{i}, t_{i}\right)=\left(\bar{\lambda}^{(i)} t_{i}+q_{\lambda}\right)^{-q_{\lambda}}\left(\frac{\bar{\lambda}^{(i)} t_{i}}{\bar{\lambda}^{(i)} t_{i}+q_{\lambda}}\right)^{n_{i}},
$$

in which $\bar{\lambda}^{(i)}$ is the mean earthquake activity rate, corresponding to the magnitude level of completeness $m_{\min }^{(i)}$, and is defined as

$$
\bar{\lambda}^{(i)}=\bar{\lambda}\left[1-F_{M}\left(m_{\min }^{(i)} \mid v_{\beta}, m_{\min }\right)\right]
$$

with $F_{M}(\cdot \mid \cdot)$ as defined in (13). Following the definition of the likelihood function, based on a set of independent observations and (15), the likelihood function $L_{i}\left(\bar{\beta} \mid \mathbf{m}_{i}\right)$ takes the form:

$$
L_{i}\left(\bar{\beta} \mid \mathbf{m}_{i}\right)=\left[C_{\beta} \bar{\beta}\right]^{n_{i}} \prod_{k=1}^{n_{i}}\left[1+\frac{\bar{\beta}}{q_{\beta}}\left(m_{i k}-m_{\min }^{(i)}\right)\right]^{-\left(q_{\beta}+1\right)} .
$$

Equations (20)-(22) define the likelihood function of the unknown parameters $\boldsymbol{\theta}=\left(\bar{\lambda}, \bar{\beta}, m_{\max }\right)$ for each complete subcatalog. The likelihood function, based on all $s$ complete subcatalogs takes the form:

$$
L_{\mathrm{C}}(\boldsymbol{\theta})=\prod_{i=1}^{s} L_{i}\left(\bar{\lambda} \mid n_{i}, t_{i}\right) L_{i}\left(\bar{\beta} \mid \mathbf{m}_{i}\right)
$$

\section{Combining Historical and Complete Catalogs}

Finally, $L(\boldsymbol{\theta})$ is the joint likelihood function based on all the available data and is calculated as the product of the likelihood functions, based on the historical and complete parts of the catalog, as

$$
L(\boldsymbol{\theta})=L_{H}(\boldsymbol{\theta}) \times L_{\mathrm{C}}(\boldsymbol{\theta}) .
$$

The maximum likelihood procedure is used to derive estimates for the parameters $\bar{\lambda}$ and $\bar{\beta}$, which, for a given maximum area-characteristic earthquake magnitude $m_{\max }$, maximizes the likelihood function $L(\boldsymbol{\theta})$. The maximum of the likelihood function is obtained by solving the system of two equations $\frac{\partial \ell}{\partial \bar{\lambda}}=0$ and $\frac{\partial \ell}{\partial \bar{\beta}}=0$, in which $\ell=\ln [L(\boldsymbol{\theta})]$. A variance-covariance matrix $\mathbf{D}(\boldsymbol{\theta})$ of the estimated $\hat{\bar{\lambda}}$ and $\hat{\bar{\beta}}$ is calculated according to the formula by Edwards (1972):

$$
\mathbf{D}(\hat{\bar{\lambda}}, \hat{\bar{\beta}})=-\left[\begin{array}{cc}
\frac{\partial^{2} \ell}{\partial \bar{\lambda}^{2}} & \frac{\partial^{2} \ell}{\partial \bar{\lambda} \partial \bar{\beta}} \\
\frac{\partial^{2} \ell}{\partial \bar{\beta} \partial \bar{\lambda}} & \frac{\partial^{2} \ell}{\partial \tilde{\beta}^{2}}
\end{array}\right]^{-1}
$$

in which derivatives are calculated at the point $\bar{\lambda}=\hat{\bar{\lambda}}$ and $\bar{\beta}=\hat{\bar{\beta}}$.
Estimation of $m_{\text {max }}$

The next step is to estimate the maximum possible earthquake magnitude $m_{\max }$. Unfortunately, the proposed sample likelihood function $L(\boldsymbol{\theta})$ is constructed in such a way that the range of magnitudes depends on the one unknown parameter $m_{\text {max }}$, which violates the condition of regularity (LeCam, 1970; Cheng and Traylor, 1995). The resulting likelihood function therefore reaches its maximum at the maximum observed earthquake magnitude $m_{\mathrm{max}}^{\mathrm{obs}}$ and not at the required maximum possible magnitude $m_{\max }$. Consequently, the unbiased value of $m_{\max }$ cannot be estimated by applying the standard maximum likelihood procedure. A more realistic estimation of $m_{\max }$ can be provided by introducing additional information (Pisarenko, 1991; Pisarenko et al., 1996), such as the condition that the largest observed earthquake magnitude $m_{\max }^{\mathrm{obs}}$, within the span of the entire earthquake catalog, is equal to the largest expected earthquake magnitude $E\left[m_{\max }^{\text {obs }} ; t\right]$. As shown in Kijko (2004) and Kijko and Singh (2011), the introduction of such a condition leads to

$$
m_{\max }=m_{\max }^{\mathrm{obs}}+\int_{m_{\min }}^{m_{\max }}\left[F_{M}(\zeta)\right]^{n} d \zeta
$$

in which $F_{M}(\zeta)$ denotes the compound CDF of earthquake magnitude (13). Unfortunately, the integral in (25) does not have a simple solution, but an approximate assessment can be obtained through the application of the method introduced by Cramér (1961). For large $n(n>10)$, the value of $\left[F_{M}(\zeta)\right]^{n}$ is approximately equal to $\exp \left\{-n\left[1-F_{M}(\zeta)\right]\right\}$. After the substitution of the Cramér's approximation into (25), $m_{\max }$ takes the form (Kijko, 2004):

$m_{\max }=m_{\max }^{\mathrm{obs}}+\frac{\delta^{\frac{1}{q}} \exp \left[\frac{n r^{q}}{\left(1-r^{q}\right)}\right]}{\bar{\beta}}\left[\Gamma\left(-\frac{1}{q}, \delta r^{q}\right)-\Gamma\left(-\frac{1}{q}, \delta\right)\right]$,

in which

$$
\begin{aligned}
& r=\frac{p_{\beta}}{p_{\beta}+m_{\max }-m_{\max }}, c_{1}=\exp \left[-n\left(1-C_{\beta}\right)\right], \\
& \delta=n C_{\beta}, p_{\beta}=\bar{\beta} / \sigma_{\beta}^{2},
\end{aligned}
$$

and $\Gamma(\cdot, \cdot)$ is the complementary incomplete gamma function (Abramowitz and Stegun, 1970). Because the unknown $m_{\max }$ is present in both sides of equation (26), the estimator for $m_{\max }$ can be calculated only through an iteration process. The approximate variance of the $m_{\max }$ estimator subsequently assumes the form:

$$
\begin{aligned}
& \operatorname{Var}\left[m_{\max }\right]=\sigma_{M}^{2} \\
& \quad+\left[\frac{\delta^{\frac{1}{q}} \exp \left[\frac{n r^{q}}{\left(1-r^{q}\right)}\right]}{\bar{\beta}}\left[\Gamma\left(-\frac{1}{q}, \delta r^{q}\right)-\Gamma\left(-\frac{1}{q}, \delta\right)\right]\right]^{2},
\end{aligned}
$$


in which $\sigma_{M}$ denotes the standard error in the determination of the largest observed magnitude $m_{\max }^{\mathrm{obs}}$.

The maximization of the sample likelihood function $L(\boldsymbol{\theta})$, together with (26), provides the maximum likelihood estimates of the area-characteristic seismic-hazard parameters $\bar{\lambda}, \bar{\beta}$, and $m_{\max }$. These equations are solved iteratively.

It has to be noted that the above-described procedure for the assessment of the area-characteristic maximum possible earthquake magnitude $m_{\max }$ can provide an underestimated value, especially when the applied earthquake catalog is short (Kijko, 2004). In essence, improvements to the $m_{\max }$ estimation procedure can be done in two different ways. In the first approach, the bias in the $m_{\max }$ estimation is assessed and removed (Lasocki and Urban, 2011). The second approach (Cornell, 1994) utilizes a Bayesian formalism, which allows the combination of all the available information sources. These additional sources include seismic-event catalogs, information on the regional and local geology and tectonics, prehistoric earthquakes, the activity of tectonic faults, seismic history of similar regions, and/or a database of $m_{\max }$ for different seismogenic regions. The Bayesian procedure of Cornell (1994) unfortunately has a flaw that results in a biased estimate of $\hat{m}_{\max }$. The degree and sign of the bias depend on the applied estimation technique. Kijko (2012) showed that if the maximum posterior estimate was used, the bias would be negative, and the resulting underestimation of $m_{\max }$ could be as large as 0.5 units of magnitude.

\section{Accounting for Uncertainty in Earthquake Magnitude Determination}

By definition, it is impossible to determine earthquake magnitude without error. At best, the magnitudes are determined with an accuracy of up to 0.1 magnitude units, but this accuracy is rarely better than 0.2 units, especially for historical events. In some instances, the uncertainty in the magnitude of historical events can reach up to 0.5 units of magnitude. In KS-II, two models of earthquake magnitude uncertainty are considered, namely the Hard Bounds Model and the Soft Bounds Model. In the Hard Bounds Model, the earthquake magnitude is defined by both the lower and the upper magnitude limits, and it is assumed that this interval contains the real, unknown magnitude. The Soft Bounds Model defines the uncertainty of earthquake magnitude as the departure of the observed magnitude from the true, unknown value, and it is assumed that such departure follows a Gaussian distribution. The methodology implemented here will refer only to the Soft Bound Model. Despite the complexity of implementing this model, it is undoubtedly the preferred model to account for magnitude uncertainty (Tinti and Mulargia, 1985; Rhoades, 1996; Rhoades and Dowrick, 2000; Marzocchi and Sandri, 2003; Márquez-Ramírez et al., 2015).

Utilizing the Soft Bound Model, if the error of magnitude determination followed a Gaussian distribution with a zero mean and a standard deviation $\sigma_{M}$, the CDF of the observed magnitude would become (Tinti and Mulargia, 1985):
$G_{M}(m)=\int_{m_{\min }}^{m_{\max }} f_{M}(\zeta)\left[\int_{-\infty}^{m-\zeta} \frac{1}{\sigma_{M} \sqrt{2 \pi}} \exp \left(-\frac{\varepsilon^{2}}{2 \sigma_{M}^{2}}\right) \mathrm{d} \varepsilon\right] \mathrm{d} \zeta$

in which the PDF $f_{M}(\zeta)$ denotes the compound exponentialgamma magnitude distribution (15). Obtaining the closedform expression of the CDF (28) and the corresponding PDF is no trivial task. It can be shown (S. Verryn, personal comm., 2011) that the CDF (28) can be approximated by the formula:

$$
\begin{gathered}
G_{M}(m)=\frac{C_{\beta} \bar{\beta} q_{\beta}^{q+1}}{2 \sigma_{M}}\{A+B\}, \\
A=\left.\frac{\left(r_{1}+r_{2} \alpha\right)^{-q_{\beta}}}{r_{2} q_{\beta}}\right|_{\frac{m-m_{\min }}{\sigma_{M}}} ^{\frac{m-m_{\max }}{\sigma_{M}}}, \\
B=\left(\frac{2}{\pi}\right)^{1 / 2} \sum_{h=0}^{\infty} \frac{(-1)^{h}}{2^{h} h !(2 h+1)} \frac{1}{b^{w+1}} \\
\times \sum_{j=0}^{2 h+1} \frac{w !\left(-r_{1}\right)^{j}\left(r_{1}+r_{2} \alpha\right)^{w-q_{\beta}-j}}{(w-j) ! j !\left(w-q_{\beta}-j\right)} \mid \begin{array}{l}
\frac{m-m_{\max }}{\sigma_{M}} \\
\frac{m-m_{\min }}{\sigma_{M}}
\end{array}
\end{gathered}
$$

in which $a=q_{\beta}+\bar{\beta}\left(m-m_{\min }\right)$ and $b=-\bar{\beta} \sigma_{M}$. Please refer to the Appendix for the derivation of (29).

\section{Special Instances}

At times, seismic-event catalogs contain only one type of dataset; namely either historical or complete (Fig. 1). In this section, the special instances are further investigated, such as (a) the seismic-event catalog contains information only about historical events, and (b) the seismic-event catalog consists only of complete subcatalogs at different levels of completeness.

\section{Only Historical Earthquake Catalogs Are Available}

To provide a reliable assessment of the seismic hazard for a specified region, observations spanning hundreds of years are required. For many earthquake-threatened areas, only historical records are available; however, these are incomplete and contain information only about the largest and most catastrophic earthquake occurrences. In such an instance, the sample likelihood function $L(\boldsymbol{\theta})$ takes the familiar form (19). The proposed procedure can be further simplified by replacing the compound Poisson-gamma distribution (13) with the classic Poisson distribution (1) and replacing the compound exponential-gamma distribution of the earthquake magnitude (13) with the equivalent classic GutenbergRichter distribution (3), truncated from the top.

It is shown that after the replacement of the compound distributions by their classic counterparts, the solution of the 
system of equations $\frac{\partial \ell}{\partial \bar{\lambda}}=0$ and $\frac{\partial \ell}{\partial \bar{\beta}}=0$ is obtained as (Kijko and Dessokey, 1987; KS-I):

$$
\left\{\begin{array}{l}
\frac{1}{\lambda}=\frac{\overline{m_{0}}-\overline{t_{0} \mathbf{A}}}{A_{2}-A_{1}} \\
\frac{1}{\beta}=\frac{\overline{t_{0} m_{0} \mathbf{A}}-\overline{t_{0}} A_{2} m_{\max }}{\overline{t_{0} \mathbf{A}}-\bar{t}_{0} A_{2}},
\end{array},\right.
$$

in which $\mathbf{A}$ is a column vector, with elements $A\left(m_{0 k}\right)=$ $\exp \left(-\bar{\beta} m_{0 k}\right), k=1, \ldots, n_{0}$, and

- $\overline{m_{0}}=\sum_{k=1}^{n_{0}} m_{0 k} / n_{0}$

- $\overline{t_{0}}=\sum_{k=1}^{n_{0}} t_{0 k} / n_{0}$,

- $\overline{t_{0} \mathbf{A}}=\sum_{k=1}^{n_{0}} t_{0 k} \exp \left(x \bar{\beta} m_{0 k}\right) / n_{0}$,

- $\overline{t_{0} m_{0} \mathbf{A}}=\sum_{k=1}^{n_{0}} t_{0 k} m_{0 k} \exp \left(-\bar{\beta} m_{0 k}\right) / n_{0}$,

- $A_{1}=\exp \left(-\bar{\beta} m_{\min }\right)$

- $A_{2}=\exp \left(-\bar{\beta} m_{\max }\right)$.

For the specified value of $m_{\max }$, the solution set (30) provides the maximum likelihood estimates of the required earthquake-hazard parameters $\lambda$ and $\beta$. It is interesting to note that for $m_{\max } \rightarrow+\infty$ and $t_{0 k}=$ const, the system of equations (30) is reduced to the maximum likelihood estimation of parameters $\lambda$ and $\beta$ of the first Gumbel distribution (Kimball, 1946).

\section{Only Complete Earthquake Catalogs Are Available}

In the instance in which the historical part of the catalog is absent, the compound solutions of (23) and (24) can be significantly simplified. A simple, overall maximum likelihood estimate of $\bar{\lambda}$ and $\bar{\beta}$ value is obtained by applying the additive property of likelihood functions (Rao, 1973). When applied to the current problem, the joint likelihood function of the $\bar{\beta}$ value, which utilizes all seismic events that occurred within the span of the complete catalog, with $s$ different levels of completeness, is defined as

$$
L(\bar{\beta})=\prod_{i=1}^{s} L_{i}\left(\bar{\beta} \mid \mathbf{m}_{i}\right),
$$

in which $L_{i}\left(\bar{\beta} \mid \mathbf{m}_{i}\right)$ represents the $i$ th likelihood function (22) based on the $i$ th complete earthquake subcatalog when $i=1,2, \ldots, s$. Further simplification followed by replacing the compound earthquake magnitude distribution (18) with the classic Gutenberg-Richter CDF (3), truncated from the top. The maximization of (31) provides an estimator of $\bar{\beta}$ in the form (Kijko and Smit, 2012)

$$
\hat{\beta}=\left(\frac{r_{1}}{\hat{\beta}^{(1)}}+\frac{r_{2}}{\hat{\beta}^{(2)}}+\ldots+\frac{r_{\mathrm{s}}}{\hat{\beta}^{(s)}}\right)^{-1},
$$

in which $r_{i}=n_{i} / n, n=\sum_{i=1}^{s} n_{i}$ is the total number of seismic events in a complete catalog, with magnitudes equal to or exceeding the relevant level of completeness $m_{\text {min }}^{(i)}$, and $\hat{\beta}^{(i)}$ is defined as

$$
\hat{\beta}^{(i)}=\frac{1}{\overline{\mathbf{m}_{i}}-m_{\min }^{(i)}},
$$

such that $\overline{\mathbf{m}_{i}}=\sum_{k=1}^{n_{i}} m_{i k} / n_{i}$ denotes the mean of the earthquake magnitudes observed within the complete part of the subcatalog $i$. It is clear that (33) is the classic Aki-Utsu estimator (Aki, 1965; Utsu, 1965) of the $\beta$ values, as calculated for each of the individual complete earthquake subcatalogs. Once the $\hat{\beta}$ value is known, the mean value of the earthquake activity rate $\lambda \equiv \lambda\left(m_{\min }\right)$ is calculated. It can be shown (KS-I; Kijko and Smit, 2012) that if the number of seismic events per time unit is a Poisson random variable, the compound Poisson-gamma distribution (13) is replaced by the classic Poisson distribution (1), and the maximum likelihood estimator of $\lambda\left(m_{\min }\right)$ takes the form

$$
\hat{\lambda}\left(m_{\min }\right)=\frac{n}{\sum_{i=1}^{s} t_{i} \exp \left[-\hat{\beta}\left(m_{\min }^{(i)}-m_{\min }\right)\right]} .
$$

For a single complete earthquake catalog, in which $i=1$, $m_{\min }^{(1)}=m_{\min }^{(2)}=m_{\min }^{(s)}=m_{\min } ; t=t_{1} \quad$ with $t_{2}=t_{3}=\ldots$ $=0$, and $n=n_{1}$ with $n_{2}=n_{3}=\ldots=0$, the estimator (34) reduces to the classic maximum likelihood estimator of the Poisson distribution parameter and takes the form $n / t$.

The assessment of hazard parameters from a catalog with varying levels of completeness is no trivial task, and different solutions to the problem have been proposed (Molchan et al., 1970; Rosenblueth, 1986; Rosenblueth and Ordaz, 1987; Kijko and Smit, 2012). However, the most elegant, straightforward, and the best-known solution is the procedure derived by Weichert (1980).

\section{Example of Application}

For the purpose of illustration, the proposed methodology is applied to the area that had experienced the strongest and most devastating earthquake in contemporary South African history. The 29 September $1969 M_{\mathrm{w}} 6.3$ CeresTulbagh event occurred about $100 \mathrm{~km}$ from the major metropolitan city of Cape Town (Green and Bloch, 1971; Green and McGarr, 1972; Kijko et al., 2003; Krüger and Scherbaum, 2014). Several buildings in the area suffered serious structural damage, which varied from the almost total destruction of old and poorly constructed buildings to large cracks appearing in better-constructed infrastructure. Twelve people were killed and many more injured. This event resulted in an insured loss of US \$7.4 million at the time of occurrence; however, the uninsured loss was roughly 3.5 times higher (Davies and Kijko, 2003).

Reported seismicity in the vicinity of Cape Town dates back as far as 1620 . The seismicity is typical of an intraplate region and is characterized by low-level activity, compared with world standards. The earthquakes are randomly distributed in space and time. Perhaps the most common practice in seismic-hazard analysis is that the maximum possible earthquake magnitude is estimated from the magnitude-fault- 
Table 1

The Largest Earthquakes That Occurred within a $300 \mathrm{~km}$ Radius of the Ceres-Tulbagh Earthquake Epicenter

\begin{tabular}{clcclc}
\hline Event & \multicolumn{1}{c}{ Date } & Magnitude & Event & \multicolumn{1}{c}{ Date } & Magnitude \\
\hline 1 & 13 July 1766 & 4.3 & 15 & 30 September 1950 & 5.5 \\
2 & 4 December 1809 & 6.3 & 16 & 13 June 1951 & 4.7 \\
3 & 2 June 1811 & 5.7 & 17 & 28 January 1952 & 5.4 \\
4 & 14 April 1819 & 4.3 & 18 & 26 February 1953 & 4.4 \\
5 & 11 November 1835 & 4.3 & 19 & 30 September 1957 & 4.2 \\
6 & 14 August 1857 & 5.0 & 20 & 29 August 1960 & 4.8 \\
7 & 13 September 1899 & 5.0 & 21 & 27 August 1963 & 5.0 \\
8 & 28 May 1902 & 4.3 & 22 & 21 February 1964 & 4.3 \\
9 & 30 December 1908 & 4.0 & 23 & 28 September 1965 & 4.3 \\
10 & 6 July 1911 & 4.0 & 24 & 1 March 1966 & 4.3 \\
11 & 9 October 1921 & 5.0 & 25 & 16 June 1967 & 4.3 \\
12 & 11 August 1926 & 4.0 & 26 & 29 September 1969 & 6.3 \\
13 & 13 October 1940 & 4.3 & 27 & 14 April 1970 & 5.7 \\
14 & 23 October 1941 & 4.3 & & & \\
\end{tabular}

The catalog starts on 1 January 1751 and ends on 31 December 1970.

Table 2

A Summary of the Complete Parts of the Catalogs

\begin{tabular}{cccccc}
\hline & & & & & \\
Subcatalog & Start Date & End Date & Level of Completeness $\left(M_{\mathrm{w}}\right)$ & Number of Events & $\begin{array}{c}\text { Standard Error of Earthquake } \\
\text { Magnitude Determination }\end{array}$ \\
\hline 1 & 1 January 1971 & 31 December 1990 & 4.0 & 7 & 0.3 \\
2 & 1 January 1991 & 31 December 1995 & 3.5 & 2 & 0.2 \\
3 & 1 January 1996 & 31 January 2013 & 3.0 & 29 & 0.1 \\
\hline
\end{tabular}

length relationships (Wells and Coppersmith, 1994; Leonard, 2010; Stirling et al., 2013). However, the correlation between most of the observed earthquakes in South Africa and the surface expression of major geological features is generally not clear (Fernandez and Guzman, 1979a,b; Brandt et al., 2005). The estimated area-characteristic maximum possible earthquake magnitude is therefore calculated by utilizing the observed seismic-event catalog.

The seismic-event database for South Africa, especially the historical catalog, is highly incomplete because large parts of the country are sparsely populated, and the detection capabilities of the seismic network are anything but uniform (Saunders et al., 2008). The seismic-event catalog used in this study was compiled from several sources. After a critical analysis of each of the data sources, the main contribution to pre-instrumentally recorded seismicity was that of Brandt et al. (2002). The instrumentally recorded events were selected mainly from the available databases provided by the Council for Geoscience, Pretoria, South Africa, and the International Seismological Centre in Edinburgh, Scotland. For illustrative purposes, the catalog used in the present analysis spans the period 1 January 1751 to 31 January 2012 (see Data and Resources). The events were selected from within a circle, with radius of $300 \mathrm{~km}$ from the anticipated epicenter, which is the Ceres-Tulbagh earthquake (33.28'S, $19.70^{\prime} \mathrm{E}$ ) (Kijko et al., 2003). The seismic-event catalog was divided into an incomplete historical part, consisting of only the largest events (Table 1), and the complete instrumental part. It was assumed that earthquake magnitudes for the incomplete part of the catalog were determined with a standard error equal to 0.3 magnitude units. The complete part of the catalog was further divided into three subcatalogs, each with different levels of completeness and assumed magnitude standard errors of $0.3,0.2$, and 0.1 (Table 2). Based on several tests on the seismicity of the selected area, it was assumed that the uncertainty of the earthquake-occurrence model parameters was equal to $25 \%$. This implied that the space-time variation of parameters $\lambda$ and $\beta$, relative to their mean values, was equal to 0.25 . Figure 2 a depicts the mean return periods, and Figure $2 \mathrm{~b}$ depicts the probability that a given magnitude would be exceeded at least once in any year for the selected area, as estimated by the proposed procedure (KSS-III). Each graph also provides the calculated level of confidence for the calculated values.

To capture the effect of incorporated uncertainties in both the magnitude determination and the selected earthquakeoccurrence model, two different comparisons were made. In the first comparison (Fig. 3), the differences were investigated between the estimates of the mean return periods according to KS-I (magnitude and model uncertainties were ignored) and two respective alternative instances. These alternative instances are (1) only the uncertainty of the earthquake magni- 


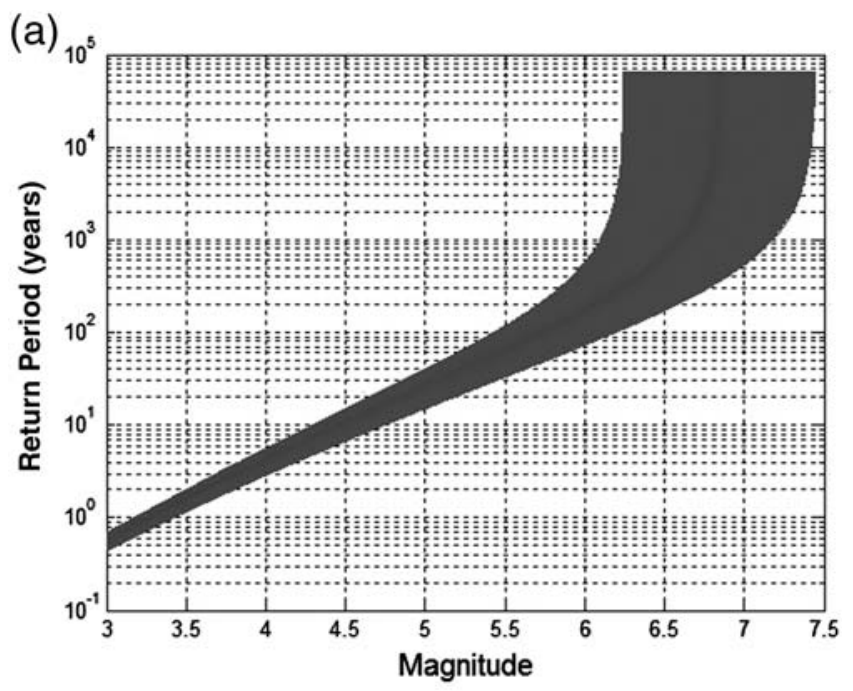

(b)

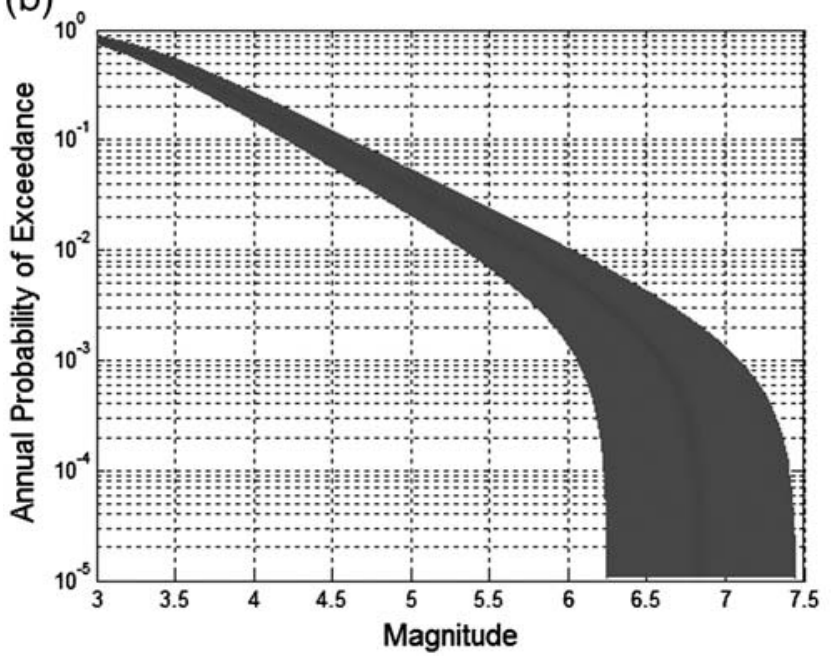

Figure 2. (a) The mean return periods and (b) the probability that a given magnitude will be exceeded at least once in any year. Each graph also provides the calculated level of confidence for the calculated values.

tude is taken into account (KS-II), and (2) only the uncertainty of the earthquake-occurrence model is considered. The purpose of such a comparison was to isolate and capture the effects of the two uncertainties.

The comparison in Figure 3 of the classic instance (KS-I), with the instance in which only magnitude uncertainties were taken into account, confirms the acknowledged fact that accounting for magnitude errors leads to an increase of return periods or, equivalently, to a decrease of seismic hazard (e.g., Tinti and Mulargia, 1985; Rhoades and Dowrick, 2000; McGuire, 2004). The uncertainty of the earthquake-occurrence model has an opposite effect, namely it leads to a decrease in the return periods or, equivalently, an increase in seismic hazard.

The second comparison (Fig. 4) shows two estimates of the mean return periods according to KS-I, when both magnitude and model uncertainties are ignored; and the same

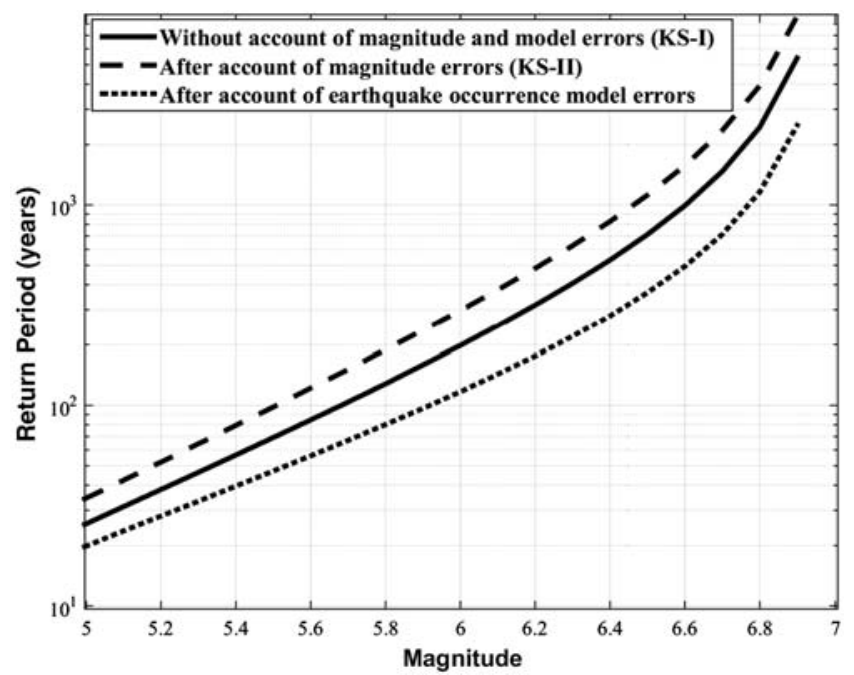

Figure 3. The comparison between the individual effects of the magnitude uncertainties and the event-occurrence model uncertainties on the mean return periods. The solid line shows the classic instance (Kijko and Sellevoll, 1989 [KS-I]), when both the uncertainties are ignored. The dashed line captures the effect of magnitude uncertainties (procedure KS-II), whereas the dotted line shows the effect of accounting for the model uncertainties.

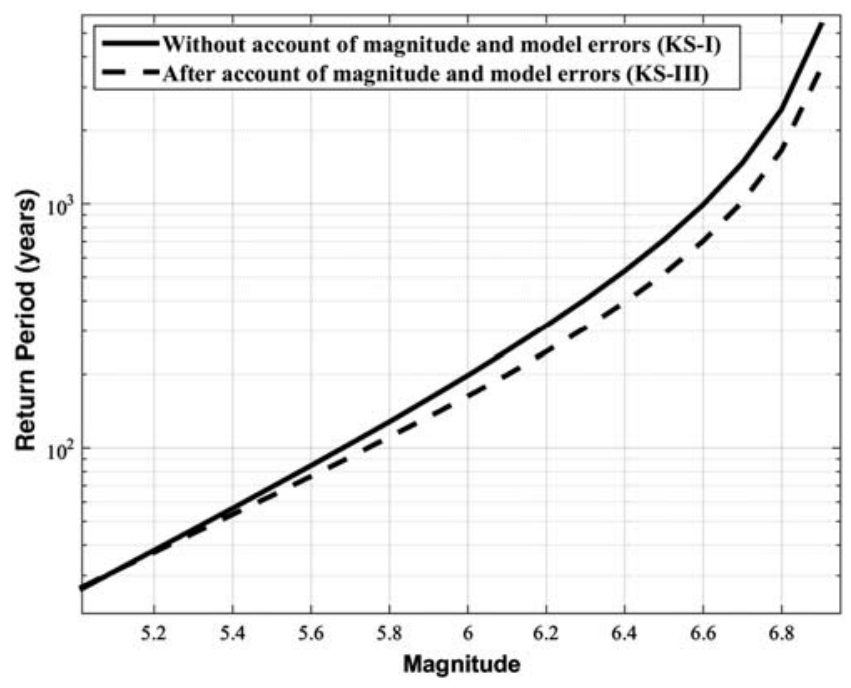

Figure 4. The comparison of mean return periods calculated without accounting for magnitude and event-occurrence model uncertainties (the solid line), with return periods calculated taking into account the magnitude and model uncertainties (the dashed line). The dashed line is a balance of the two opposite effects of the modeled uncertainties.

estimates as those obtained by KSS-III, when both the magnitude and the event-occurrence model uncertainties are taken into account. The increase of return periods, as a result of magnitude uncertainty, and the decrease of return periods, as an effect of the uncertainty of the earthquake-occurrence model, averages out to an overall decrease in return periods. Consequently, the calculated hazard levels increase, as the 
effect of introducing model uncertainty is stronger than the effect of magnitude uncertainty.

\section{Remarks and Conclusions}

A methodology is presented to assess the area-characteristic seismic-hazard parameters; the mean seismic activity rate of $\lambda$ and the $b$-value of Gutenberg-Richter, based on incomplete catalogs; the uncertainty in the earthquake magnitude determination; as well as the uncertainty associated with the applied earthquake-occurrence models. The present work is a natural expansion of the work by Kijko and Sellevoll $(1989,1992)$ to develop efficient techniques for the optimal assessment of the area-characteristic seismic-hazard parameters by including uncertainty in the earthquake-occurrence model. The current approach extends the classic earthquakeoccurrence model, the frequency-magnitude GutenbergRichter relation, and the Poisson distribution by the introduction of their compounded counterparts.

The newly derived procedure was applied to the area in the vicinity of Cape Town that had experienced an event of magnitude 6.3 on 29 September 1969, one of the strongest and most devastating earthquakes in the history of South Africa. The present study confirms the recognized fact (Tinti and Mulargia, 1985; Rhoades and Dowrick, 2000; McGuire, 2004) that accounting for magnitude uncertainty leads to an increase of return periods or, equivalently, to a decrease of seismic hazard. The uncertainty of the earthquake-occurrence model has the opposite effect, namely it leads to a decrease in return periods and a subsequent increase of seismic hazard.

Several additional aspects should be investigated in the future. These include (1) a more comprehensive investigation of the universal applicability of the above results to seismichazard modeling in areas with different levels of seismic activity, (2) the sensitivity of the hazard parameters to different degrees of model uncertainty, (3) the effect of different distribution models of magnitude uncertainty, and (4) the relationship between magnitude uncertainty and uncertainty in the earthquake-occurrence models.

\section{Data and Resources}

The instrumentally recorded events were mainly selected from the available database provided by the Council for Geoscience, Pretoria, South Africa, and the International Seismological Centre in the United Kingdom (http://www. isc.ac.uk, last accessed May 2015). The catalog used in the analysis spans a period of $\sim 268$ years, namely from 1 January 1751 to 31 January 2012. The MATLAB computer program (www.mathworks.com/products/matlab, last accessed March 2016), used for the calculation of the newly derived estimator of the mean annual activity rate $\lambda$, the $b$-value of the Gutenberg-Richter magnitude-frequency relation, and the area-characteristic maximum possible earthquake magnitude $m_{\max }$ is available for academic purposes from the authors. Following the requirements expressed by Budnitz et al. (1997), the computer code also contains the procedure for the calculation of the correlation coefficient between $\lambda$ and the $b$-value.

\section{Acknowledgments}

The authors sincerely thank Sandra Verryn for providing the derivation of exponential-gamma distribution of apparent magnitude (equation 30). This work is based on the research supported wholly/in part by the National Research Foundation of South Africa (Grant Numbers 76906 and 94808).

\section{References}

Abramowitz, M., and I. A. Stegun (1970). Handbook of Mathematical Functions, Ninth Ed., Dover, New York.

Aki, K. (1965). Maximum likelihood estimate of $b$ in the formula $\log N=$ $a-b M$ and its confidence limits, Bull. Earthq. Res. Inst. Tokyo Univ. 43, 237-239.

Benjamin, J. R. (1968). Probabilistic models for seismic forces design, J. Struct. Div. ASCE 94, (ST5), 1175-1196.

Brandt, M. B. C., M. Bejaichund, E. M. Kgaswane, E. Hattingh, and D. L. Roblin (2005). Seismic History of South Africa, Council for Geoscience, Seismological Series, 37, 32 pp.

Brandt, M. B. C., I. Saunders, and G. Graham (2002). Frequency-magnitude relationship and estimated from micro-seismic data, and regional- and historical data sets for the southern part of South Africa, Africa, Geosci. Rev. 9, no. 3, 199-210.

Budnitz, R. J., G. Apostolakis, D. M. Boore, L. S. Cluff, K. J. Coppersmith, C. A. Cornell, and P. A. Morris (1997). Recommendations for Probabilistic Seismic Hazard Analysis: Guidance on Uncertainty and Use of Experts, NUREG/CR-6372, UCR-ID-122160, Main Rept. 1, Prepared for Lawrence Livermore National Laboratory, $63 \mathrm{pp}$.

Campbell, K. W. (1982). Bayesian analysis of extreme earthquake occurrences, part I: Probabilistic hazard model, Bull. Seismol. Soc. Am. 72, 1689-1705.

Campbell, K. W. (1983). Bayesian analysis of extreme earthquake occurrences, part II: Application to the San Jacinto fault zone of southern California, Bull. Seismol. Soc. Am. 73, 1099-1115.

Cheng, R. H., and L. Traylor (1995). Non-regular maximum likelihood problems, J. Roy. Stat. Soc.B 57, 3-44.

Cornell, C. A. (1968). Engineering seismic risk analysis, Bull. Seismol. Soc. Am. 58, 1583-1606.

Cornell, C. A. (1994). Statistical analysis of maximum magnitudes in the earthquakes of stable continental regions, in The Earthquakes of Stable Continental Regions. Assessment of Large Earthquake Potential, J. Schneider (Editor), Vol. 1, EPRI, Palo Alto, California, NP-4726, $5-1-5-27$

Cornell, C. A., and S. R. Winterstein (1988). Temporal and magnitude dependence in earthquake recurrence models, Bull. Seismol. Soc. Am. 78, 1522-1537.

Cramér, H. (1961). Mathematical Methods of Statistics, Princeton University Press, Princeton, New Jersey.

Davies, N., and A. Kijko (2003). Seismic risk assessment: With an application to the South African insurance industry, S. Afr. Actuarial J. $3,1-28$.

DeGroot, M. H. (1970). Optimal Statistical Decisions, McGraw-Hill, New York, New York.

Edwards, A. W. F. (1972). Likelihood, Cambridge University Press, New York, New York, $235 \mathrm{pp}$.

Epstein, B., and C. Lomnitz (1966). A model for occurrence of large earthquakes, Nature 211, 954-956.

Fernandez, L. M., and J. A. Guzman (1979a). Earthquake hazard in southern Africa, Geological Survey of South Africa, Vol. 10, Seismologic Series, $22 \mathrm{pp}$.

Fernandez, L. M., and J. A. Guzman (1979b). Seismic history of southern Africa, Geol. Surv. S. Afr. Seismologic Series, 9, 1-38. 
Gan, Z. J., and C. C. Tung (1983). Extreme value distribution of earthquake magnitude, Phys. Earth Planet. Inter. 32, 325-330.

Gardner, J. K., and L. Knopoff (1974). Is the sequence of earthquakes in southern California, with aftershocks removed, Poissonian? Bull. Seismol. Soc. Am. 64, 1363-1368.

Gibowicz, S. J., and A. Kijko (1994). An introduction to mining seismology, Academic Press, San Diego, California, 396 pp.

Green, R. W. E., and S. Bloch (1971). The Ceres, South Africa, earthquake of September 29, 1969. I. Report on some aftershocks, Bull. Seismol. Soc. Am. 61, no. 4, 851-859.

Green, R. W. E., and A. McGarr (1972). A comparison of the focal mechanism and aftershock distribution of the Ceres, South Africa, earthquake of September 29, 1969, Bull. Seismol. Soc. Am. 62, no. 3, 869-871.

Gutenberg, B., and C. F. Richter (1942). Earthquake magnitude, intensity, energy, and acceleration, Bull. Seismol. Soc. Am. 32, 163-191.

Gutenberg, B., and C. F. Richter (1956). Earthquake magnitude, intensity, energy, and acceleration: (Second paper), Bull. Seismol. Soc. Am. 46, $105-145$.

Guttorop, P., and D. Hopkins (1986). On estimating varying $b$ values, Bull. Seismol. Soc. Am. 76, no. 3, 889-895.

Hamada, M. S., A. G. Wilson, C. Shane Reese, and H. F. Martz (2008). Bayesian Reliability, Springer, New York, New York, 430 pp.

Karakaisis, G. F., C. B. Papazachos, E. M. Scordilis, C. A. Papaioannou, and B. C. Papazachos (2002). Time Variation of Seismicity and Seismic Hazard in Hellenic Arc Trench System, XXXVIII General Assembly of ESC, Genoa, Italy, 1-6 September 2002.

Kijko, A. (2004). Estimation of the maximum earthquake magnitude, Pure Appl. Geophys. 161, 1-27.

Kijko, A. (2012). On Bayesian procedure for maximum earthquake magnitude estimation, Res. Geophys. 2, 1, doi: 10.4081/rg.2012.e7.

Kijko, A., and M. M. Dessokey (1987). Application of the extreme magnitude distribution to incomplete earthquake files, Bull. Seismol. Soc. Am. 77, 1429-1436.

Kijko, A., and G. Graham (1998). "Parametric-historic" procedure for probabilistic seismic hazard analysis, part I: Assessment of maximum regional magnitude, Pure Appl. Geophys. 152, 413-442.

Kijko, A., and M. A. Sellevoll (1989). Estimation of earthquake hazard parameters from incomplete data files, part I: Utilization of extreme and complete catalogues with different threshold magnitudes, Bull. Seismol. Soc. Am. 79, 645-654.

Kijko, A., and M. A. Sellevoll (1992). Estimation of earthquake hazard parameters from incomplete data files, part II: Incorporation of magnitude heterogeneity, Bull. Seismol. Soc. Am. 82, 120-134.

Kijko, A., and M. Singh (2011). Statistical tools for maximum possible earthquake magnitude estimation, Acta Geophys. 59, 674-700.

Kijko, A., and A. Smit (2012). Extension of the Aki-Utsu $b$-value estimator for incomplete catalogs, Bull. Seismol. Soc. Am. 102, 1283-1287.

Kijko, A., S. J. P. Retief, and G. Graham (2003). Seismic hazard and risk assessment for Tulbagh, South Africa, part II: Assessment of seismic risk, Nat. Hazards 30, 25-41.

Kimball, B. F. (1946). Sufficient statistical estimation functions for the parameters of the distribution of maximum values, Ann. Math. Stat. 17, 299-306.

Klugman, S. A., H. H. Panjer, and G. E. Willmot (2008). Loss models. From data to decisions, John Willey \& Sons, Inc., Hoboken, New Jersey, $726 \mathrm{pp}$.

Krüger, F., and F. Scherbaum (2014). The 29 September 1969, Ceres, South Africa, earthquake: Full waveform moment tensor inversion for point source and kinematic source parameters, Bull. Seismol. Soc. Am. 104, $576-581$.

Lasocki, S., and P. Urban (2011). Bias, variance and computational properties of Kijko's estimators of the upper limit of magnitude distribution, $M_{\max }$, Acta Geophys. 59, 659-673.

LeCam, L. (1970). On the assumptions used to prove asymptotic normality of maximum likelihood estimates, Ann. Statist. 41, 802-828.
Leonard, M. (2010). Earthquake fault scaling: Self-consistent relating of rupture length, width, average displacement, and moment release, Bull. Seismol. Soc. Am. 100, 1971-1988.

Lomnitz, C. (1973). Poisson processes in earthquake studies, Bull. Seismol. Soc. Am. 63, 735-758.

Márquez-Ramírez, V. H., F. A. Nava, and F. R. Zúñiga (2015). Correcting the Gutenberg-Richter $b$-value for effects of rounding and noise, Earthq. Sci. 28, no. 2, 129-134.

Marzocchi, W., and L. Sandri (2003). A review and new insights on the estimation of the $b$-value and its uncertainty, Ann. Geophys. 46, no. $6,1271-1282$.

McGuire, R. K. (1976). FORTRAN computer program for seismic risk analysis, U.S. Geol. Surv. Open-File Rept. 76-67.

McGuire, R. K. (1978). FRISK: Computer program for seismic risk analysis using faults as earthquake sources, U.S. Geol. Surv. Open-File Rept. 78-1007.

McGuire, R. K. (2004). Seismic Hazard and Risk Analysis, Earthquake Engineering Research Institute, Oakland, California, MNO-10, $221 \mathrm{pp}$.

Molchan, G. M., V. L. Keilis-Borok, and V. Vilkovich (1970). Seismicity and principal seismic effects, Geophys. J. 21, 323-335.

Mora, P., M. Matsu'ura, R. Madariaga, and J.-B. Minster (Editors) (2001). Microscopic and macroscopic simulation: Towards predictive modelling of the earthquake process, Pure Appl. Geophys. 157, 1817-2283.

Ogata, Y. (1999). Seismicity analysis point-process modeling: A review, Pure Appl. Geophys. 155, 471-507.

Ogata, Y., and K. Abe (1991). Some statistical features of the long-term variation of the global and regional seismic activity, Int. Stat. Rev. 59, 139-161.

Pisarenko, V. F. (1991). Statistical evaluation of maximum possible magnitude, Izvestiya, Earth Phys. 27, 757-763.

Pisarenko, V. F., and A. A. Lyubushin (1997). Statistical estimation of maximum peak ground acceleration at a given point of a seismic region, $J$. Seismol. 1, 395-405.

Pisarenko, V. F., A. A. Lyubushin, V. B. Lysenko, and T. V. Golubeva (1996). Statistical estimation of seismic hazard parameters: Maximum possible magnitude and related parameters, Bull. Seismol. Soc. Am. 86, no. 3, 691-700.

Rao, C. R. (1973). Linear Statistical Inference and Its Application, Second Ed, John Willey and Sons, New York, New York, 625 pp.

Rhoades, D. A. (1996). Estimation of the Gutenberg-Richter relation allowing for individual earthquake magnitude uncertainties, Tectonophysics 25, 871-883.

Rhoades, D. A., and D. J. Dowrick (2000). Effects of magnitude uncertainties on seismic hazard estimates, 12WCEE 2000: 12th World Conference on Earthquake Engineering, Auckland, New Zealand, 30 January to 4 February 2000.

Rosenblueth, E. (1986). Use of statistical data in assessing local seismicity, Earthq. Eng. Struct. Dynam. 14, 325-337.

Rosenblueth, E., and M. Ordaz (1987). Use of seismic data from similar regions, Earthq. Eng. Struct. Dynam. 15, 619-634.

Rotondi, R., and E. Varini (2007). Bayesian inference of stress release models applied to some Italian seismogenic zones, Geophys. J. Int. 169, 301-314.

Saunders, I., M. Brandt, J. Steyn, D. Roblin, and A. Kijko (2008). The South African national seismograph network, Seismol. Res. Lett. 792, 203-210.

Scholz, C. H. (2015). On the stress dependence of the earthquake $b$ value, Geophys. Res. Lett. 42, no. 5, 1399-1402.

Sharma, S., S. Baruah, O. P. Sahu, P. K. Bora, and R. Duarah (2013). Low $b$-value prior to the Indo-Myanmar subduction zone earthquakes and precursory swarm before the May 1995 M 6.3 earthquake, J. Asian Earth Sci. 73, 176-183.

Shi, Y., and B. A. Bolt (1982). The standard error of the magnitudefrequency $b$ value, Bull. Seismol. Soc. Am. 72, 1677-1687.

Simpson, D. W., and P. G. Richards (1981). Earthquake Prediction, an International Review, Maurice Ewing series IV, D. W. Simpson and 
P. G. Richards (Editors), American Geophysical Union, Washington, D.C., 680 pp.

Stirling, M., T. Goded, K. Berryman, and N. Litchfield (2013). Selection of earthquake scaling relationships for seismic-hazard analysis, Bull. Seismol. Soc. Am. 103, 2993-3011.

Talbi, A., and F. Yamazaki (2009). A mixed model for earthquake inter-event times, J. Seismol. 10, 289-307.

Tinti, S., and F. Mulargia (1985). Effects of magnitude uncertainties in the Gutenberg-Richter frequency-magnitude law, Bull. Seismol. Soc. Am. 75, 1681-1697.

Utsu, T. (1965). A method for determining the value of $b$ in the formula $\log n=a-b M$ showing the magnitude-frequency relation for earthquakes (with English summary), Geophys. Bull. Hokkaido Univ. 13, 99-103.

Weichert, D. (1980). Estimation of the earthquake recurrence parameters for unequal observation periods for different magnitudes, Bull. Seismol. Soc. Am. 70, 1337-1346.

Wells, D. L., and K. J. Coppersmith (1994). New empirical relationships among magnitude, rupture length, rupture width, rupture area, and surface displacement, Bull. Seismol. Soc. Am. 84, 974-1002.

Wesseloo, J. (2014). Evaluation of the spatial variation of $b$-value, J. S. Afr. Inst. Min. Metall. 114, no. 10, 823-828.

Wesseloo, J., K. Woodward, and J. Pereira (2014). Grid-based analysis of seismic data, J. S. Afr. Inst. Min. Metall. 114, no. 10, 815-822.

Wyss, M., K. Shimazaki, and A. Ito (Editors) (1999). Seismicity Patterns, their statistical significance and physical meaning, Pure Appl. Geophys. 155, 203-726.

Wyss, M., K. Shimazaki, and S. Wiemer (1997). Mapping active magma chambers by $b$ values beneath the off-Ito volcano, Japan, J. Geophys. Res. 102, no. B9, 20,413-20,422.

\section{Appendix}

The derivation of cumulative distribution function (CDF) of apparent magnitude (equation 29) by S. Verryn (personal comm., 2011)

$$
G(m)=\int_{m_{\min }}^{m_{\max }} f_{M}(\zeta)\left[\int_{-\infty}^{m-\zeta} \frac{1}{\sigma_{M} \sqrt{2 \pi}} \exp \left(-\frac{\epsilon^{2}}{2 \sigma_{M}^{2}}\right) d \varepsilon\right] d \zeta
$$

can be reduced to

$$
G(m)=\int_{m_{\min }}^{m_{\max }} f_{M}(\zeta) \Phi\left(\frac{m-\zeta}{\sigma_{M}}\right) d \zeta
$$

Assume that

$$
\Phi\left(\frac{m-\zeta}{\sigma_{M}}\right)=\frac{1}{\sqrt{2 \pi}} \int_{-\infty}^{\frac{m-\zeta}{\sigma_{M}}} \exp \left(-\frac{u^{2}}{2}\right) d u
$$

in which $\alpha=\frac{m-\zeta}{\sigma_{M}}$ such that

$$
\begin{aligned}
\Phi(\alpha) & =\frac{1}{\sqrt{2 \pi}} \int_{-\infty}^{\alpha} \exp \left(-\frac{u^{2}}{2}\right) d u, \\
& =\frac{1}{2}\left\{1+e r f\left(\frac{\alpha}{\sqrt{2}}\right)\right\}, \\
& =\frac{1}{2}\left\{1+\left(\frac{2}{\pi}\right)^{1 / 2}\left[\alpha-\frac{\alpha^{3}}{2 \times 1 ! 3}+\frac{\alpha^{5}}{2^{2} \times 2 ! 5}-\frac{\alpha^{7}}{2^{3} \times 3 ! 7}+\ldots\right]\right\} .
\end{aligned}
$$

Let $\Delta=\alpha-\frac{\alpha^{3}}{2 \times 1 ! 3}+\frac{\alpha^{5}}{2^{2} \times 2 ! 5}-\frac{\alpha^{7}}{2^{3} \times 3 ! 7}+\ldots$

Then $G(m)$ can be rewritten as

$$
\begin{aligned}
G(m) & =-\frac{1}{\sigma_{M}} \int_{\frac{m-m_{\min }}{\sigma_{M}}}^{\frac{m-m_{\max }}{\sigma_{M}}} f\left(m-\alpha \sigma_{M}\right) \Phi(\alpha) d \alpha \\
& =-\frac{1}{\sigma_{\mathrm{M}}}\left(C_{\beta} \bar{\beta} q_{\beta}^{q_{\beta}+1}\right) \\
& \times \int_{\frac{m-m_{\max }}{\sigma_{M}}}^{\frac{m-m_{\min }}{\sigma_{M}}}\left(q_{\beta}+\bar{\beta}\left(m-\alpha \sigma_{M}-m_{\min }\right)\right)^{-q_{\beta}-1} \Phi(\alpha) d \alpha \\
= & -\frac{1}{\sigma}\left(C_{\beta} \bar{\beta} q_{\beta}^{q_{\beta}+1}\right) \\
& \times \int_{\frac{m-m_{\min }}{\sigma_{M}}}^{\frac{m-m_{\max }}{\sigma_{M}}}\left[\left(q_{\beta}+\bar{\beta}\left(m-\alpha \sigma_{M}-m_{\min }\right)\right)^{-q_{\beta}-1}\right] \\
& \times\left[\frac{1}{2}\left\{1+\left(\frac{2}{\pi}\right)^{1 / 2}[\Delta]\right\} d \alpha\right. \\
= & -\frac{1}{2 \sigma}\left(C_{\beta} \bar{\beta} q_{\beta}^{q_{\beta}+1}\right) \\
& \times\left\{\int_{\frac{m-m_{\min }}{\sigma_{M}}}^{\frac{m-m_{\max }}{\sigma_{M}}}\left[\left(q_{\beta}+\bar{\beta}\left(m-\alpha \sigma_{M}-m_{\min }\right)\right)^{-q_{\beta}-1}\right] d \alpha\right. \\
& \left.+\left(\frac{2}{\pi}\right)^{1 / 2} \int_{\frac{m-m_{\min }}{\sigma_{M}}}^{\frac{m-m_{\max }}{\sigma_{m}}}\left[\left(q_{\beta}+\bar{\beta}\left(m-\alpha \sigma_{M}-m_{\min }\right)\right)^{-q_{\beta}-1}\right] \Delta d \alpha\right\} \\
= & -\frac{1}{2 \sigma}\left(C_{\beta} \bar{\beta} q_{\beta}^{q_{\beta}+1}\right)\left\{\int_{\frac{m-m_{\min }}{\sigma_{M}}}^{\frac{m-m_{\max }}{\sigma_{M}}}\left(r_{1}+r_{2} \alpha\right)^{-q_{\beta}-1} d \alpha\right. \\
& \left.+\left(\frac{2}{\pi}\right)^{1 / 2} \int_{\frac{m-m_{\min }}{\sigma_{M}}}^{\frac{m-m_{\max }}{\sigma_{M}}}\left[\left(r_{1}+r_{2} \alpha\right)^{-q_{\beta}-1}\right] \Delta d \alpha\right\}, \\
& =(\alpha)
\end{aligned}
$$

in which $r_{1}=q_{\beta}+\bar{\beta}\left(m-m_{\min }\right), r_{2}=-\bar{\beta} \sigma_{M}$ and $C_{\beta}=$ $\left(1-\left(\frac{q_{\beta}}{q_{\beta}+\bar{\beta}\left(m_{\max }-m_{\min }\right)}\right)^{q}\right)^{-1}$.

Now

$$
\int \frac{\alpha^{5}}{\left(r_{1}+r_{2} \alpha\right)^{u}} d \alpha=\frac{1}{r_{2}^{w+1}} \sum_{j=0}^{w} \frac{w !\left(-r_{1}\right)^{j}\left(r_{1}+r_{2} \alpha\right)^{w-u-j+1}}{(w-j) ! j !(w-u-j+1)}
$$

therefore,

$$
\begin{aligned}
& \int_{\frac{m-m_{\min }}{\sigma_{M}}}^{\frac{m-m_{\max }}{\sigma_{M}}}\left(r_{1}+r_{2} \alpha\right)^{-q_{\beta}-1} \\
& \quad \times\left[\alpha-\frac{\alpha^{3}}{2 \times 1 ! 3}+\frac{\alpha^{5}}{2^{2} \times 2 ! 5}-\frac{\alpha^{7}}{2^{3} \times 3 ! 7}+\ldots\right] d \alpha,
\end{aligned}
$$

and 


$$
\begin{aligned}
& \int_{\frac{m-m_{\min }}{\sigma_{M}}}^{\frac{m-m_{\max }}{\sigma_{M}}}\left(r_{1}+r_{2} \alpha\right)^{-q_{\beta}-1} d \alpha=-\left.\frac{1}{r_{2} q_{\beta}}\left(r_{1}+r_{2} \alpha\right)^{-q_{\beta}}\right|_{\frac{m-m_{\min }}{\sigma_{M}}} ^{\frac{m-m_{\max }}{\sigma_{M}}} \\
& =\sum_{h=0}^{\infty} \frac{(-1)^{h}}{2^{h} \times h !(2 h+1)} \frac{1}{r_{2}^{w+1}} \\
& \times\left.\sum_{j=0}^{2 h+1} \frac{w !\left(-r_{1}\right)^{j}\left(r_{1}+r_{2} \alpha\right)^{w-q_{\beta}-j}}{(w-j) ! j !\left(w-q_{\beta}-j\right)}\right|_{\frac{m-m_{\min }}{\sigma_{M}}} ^{\frac{m-m_{\max }}{\sigma_{M}}} .
\end{aligned}
$$

The final result is

$$
\begin{aligned}
G_{M}(m)= & \frac{C_{\beta} \bar{\beta} q_{\beta}^{q+1}}{2 \sigma_{M}}\{A+B\}, \\
A= & \left.\frac{\left(r_{1}+r_{2} \alpha\right)^{-q_{\beta}}}{r_{2} q_{\beta}}\right|_{\frac{m-m_{\min }}{\sigma_{M}}} ^{\frac{m-m_{\max }}{\sigma_{M}}}, \\
B= & \left(\frac{2}{\pi}\right)^{1 / 2} \sum_{h=0}^{\infty} \frac{(-1)^{h}}{2^{h} h !(2 h+1)} \frac{1}{b^{w+1}} \\
& \times\left.\sum_{j=0}^{2 h+1} \frac{w !\left(-r_{1}\right)^{j}\left(r_{1}+r_{2} \alpha\right)^{w-q_{\beta}-j}}{(w-j) ! j !\left(w-q_{\beta}-j\right)}\right|_{\frac{m-m_{\max }}{\sigma_{M}}} ^{\frac{m-m_{\min }}{\sigma_{M}}} .
\end{aligned}
$$

Department of Geology

University of Pretoria Natural Hazard Centre

University of Pretoria

Private Bag X20

Hatfield, Pretoria

0028

South Africa

andrzej.kijko@up.ac.za

ansie.smit@up.ac.za

(A.K., A.S.)

Department of Earth Science

University of Bergen

Allegaten 41

5007 Bergen

Norway

markvard.sellevoll@broadpark.no

(M.A.S.)

Manuscript received 8 March 2016;

Published Online 24 May 2016 